\title{
Abstract
}

The epistemological study and retrospection in fundamentals of sense perception and recollection is examined to understand the foundation of Memory. This analysis is done by a few simple tests from day to day experiences. With it, the well-known EEG signal data of individual's waking, dream and deep sleep states also analyzed. The examination establishes two fundamental discoveries: 1) A "Self induced" brain wave, having content related to old term "ego", I, Me and Myself, which corresponds to "Self Awareness"; 2) Self awareness signal conversion into earlier received signal frequency. The study also determines that the human brain does not have any information of the natural composition of the physical world.

Keywords: neuro-physics; perception; memory; self-awareness; brain 


\section{Science of self awareness and memory}

\section{Introduction}

A reader would be interested to know that while reading this manuscript, the words on the page are only a reflection of light. In other words, the reader receives light from the page manuscript. This reflected light induces or stimulates neuron "spike" in the brain which re-activates the previously registered audio signals, i.e. Memory. Memory is reactivation of previously registered signals created by neuron spikes. A word, name or description of a thing already exists in Inertia in the human brain. Except a new word, for understanding of that new word, the searched meaning is again the reflecting light of the printed word, on a Dictionary page or an audio description which is then superimposed or juxtaposed with the new visual. This phenomenon of brain mechanism is examined in many disciples concerning memory and perception.

The biophysical mechanism that persistently describes the neural activities of the brain, wherein neurons connect the nervous system to the brain, spinal cord, and the peripheral ganglia. A sensory neuron when excited by electromagnetic radiation or light or sound, other induced or external stimuli emits "spike" called electro-chemical wave which signals the brain. It is a fractional-charge of electrical emission can result in neurotransmission in the brain across synapses. Normally, humans akin to assume that memory functions like recording apparatus, which is a false assumption. The molecular mechanisms essential to the induction and continuance of memory are very dynamic and consist of divergent phases covering time periods from seconds to a lifetime (Schwarzel \& Mulluer, 2006).

Recurring sub-threshold membrane potential oscillations, as neural oscillations appear beyond synaptic and action potential, which are similar to sinusoidal wave forms (Llinas \& Yarom, 1986). Sub-threshold fluctuation frequencies show a discrepancy from hardly any $\mathrm{Hz}$ to more than $40 \mathrm{~Hz}$. With respect to the $10 \mathrm{~Hz}$ physiological vibration that controls motor execution, Theta rhythm in the entorhinal cortex (Alonso \& Llinas, 1989) and gamma band action in cortical inhibitory interneurons (Llinas et al., 1991) and in thalamus neurons, are part of ongoing investigations. (Pedroarena \& Llinas, 1997). Layers V of the entorhinal cortex have been investigated (Schmitz et al., 1998; Agrawal et al., 1999; Giocomo et al., 2007; Khosrovani et al., 2007) namely, the inferior olive in vivo, the olfactory bulb (Desmaison et al., 1999) and the dorsal cochlear nucleus (Manis et al., 1999).

The optic nerve contains retinal ganglion cell axons and support cells, leaves the eye socket orbit through the optic canal, leading towards the optic chiasm, which is situated at the base of the brain underneath the hypothalamus (Colman, 2006). An axon usually transmit neuron signal, an electrical impulse away from the neuron's cell body or soma. Large numbers of axons of the optic nerve terminate in the lateral geniculate nucleus (LGN), which is the primary relay center for visual information received from the retina and it is situated inside the thalamus of the brain. (Goodale \& Milner, 2004). The optic radiation or the geniculostriate pathway is a set of axons from relay neurons in the lateral geniculate nucleus of the thalamus suppose to transmit visual information to the visual cortex.

The critical question in cognitive neuroscience is about encoding and representation of information and mental experiences. It is not clear about the neuronal changes implicated in more intricate examples of memory, mainly declarative memory that necessitates the storage of facts and events (Byrne, 2007). From basic physics point of view, all brain activity is of sub atomic phenomenon, Whether an induced electrical discharge or internal self induced electromagnetic activity, both manifest out of atomic compositions of brain matter. Fundamentally, there is no freely available signal, one of the atoms of sodium, potassium and calcium do discharge a small fraction of its own negative charge of the value of below 30 to above 50 milli eV. 


\subsection{Background}

There are about 100 billion neurons in the brain, each of which forms synapses with many other neurons. The cell fires an electrical pulse called an action potential, when the potential changes considerably. The charged atoms such as sodium, potassium and calcium direct the synaptic activity (ScienceDaily, 2011). In the human brain, the memory capacity is the ability to store and recollect information and experiences. Since last century, scientists have formulated multimodal theories on Memory. Studies of memory provide interdisciplinary link between Cognitive psychology and neuroscience. Encoding of memory involves the spiking of individual neurons induced by sensory input, which persists even after the sensory input disappears. Encoding of episodic memory involves persistent changes in molecular structures that alter synaptic transmission between neurons. The persistent spiking in working memory can enhance the synaptic and cellular changes in the encoding of episodic memory (Jensen \& Lisman, 2005; Fransen et al., 2002). In psychology, human mental health is directly connected to individual's memory which has deep traces even before childhood. Investigations on Amnesia and memory of early childhood are still carried out (Fivush \& Schwarzmueller, 1999; Simcock \& Hayne, 2000; Bauer et al., 2000; G. Robinson-Riegler \& G. Robinson-Riegler, 2012; Phelps, 2004; Clevelend \& Reese, 2008; Joseph, 2003; Morrison \& Conway, 2010).

\subsection{Research purpose}

What information of the physical world received by the brain is trans-induced or transmitted by neurons when charged? Where does the neurotransmission end and what form does the neurotransmitter register in the brain? In other words, what is the true nature of "Memory"?

\section{Research Methodology}

Individuals from normal life (not patients) were questioned several times about their recollections of condition in deep sleep and the condition between sleep and waking state. Also several electroencephalography EEG data was analyzed which was observed, again of the normal individuals. Repeated questioning on recollection of condition in deep sleep and before and after waking up does confirm the "Self induced" signal is indeed related to old term "ego" and I, Me, including denials as well. The Self Awareness brainwave signals are active from $5 \mathrm{~Hz}$ frequency and above and not before in 0 to $4 \mathrm{~Hz}$ frequencies. The Self Awareness has also a "witness" function, which then allows individual to recollect and recount. In 0 to $4 \mathrm{~Hz}$ frequency, the individual is in Deep Sleep and never narrates that condition. Other analysis of EEG data with Self Awareness signal corroborate above stated result of conversion of same signal into received signal, produced by daily life stimulus. The Self signal becomes or modifies into the signal, externally induced from the objects perceived earlier.

\section{Results}

One extraordinary observation of brain wave patterns comprises frequencies between $0 \mathrm{~Hz}$ to $40 \mathrm{~Hz}$. Internally transmitted signal called "self-induced" has pulse energy propagation from 0 to $5 \mathrm{~Hz}$ then $8-12 \mathrm{~Hz}$, further $40 \mathrm{~Hz}$ and above. From 0 to $12 \mathrm{~Hz}$ to $40 \mathrm{~Hz}$ and above appear in fully awake conditions. The "Self induced" data signals has content related to I, Me and Myself, including denials as well. "I" is "Self Awareness" though "I" is manmade audio signal within a language. The Self Awareness brainwave signals are active from $5 \mathrm{~Hz}$ frequency and above. There are 1000s of sounds in the languages spoken around the world which correspond to "I". Verily, the "Self Awareness" signal is creation of the consciousness in the womb or before. Awaking to these signals is sensitivity to the world of information caused by receptor neurons.

\subsection{Without this signal a human being will not wake up from deep sleep}

Since it is not possible to enter deep in live brain to observe the source of brain activity or thought, an uncomplicated parallel is drawn from a Movie screen mechanism. The pictures of the physical world and the 
characters in effect are only light rays projected on the screen. They are the light frequencies on the film frames captured during shooting. The light from the projector passes through the film frames and converts according to matrix of dots into those light frequencies which were received during shooting, these then in totality covering screen appear as images and action. Similarly, the data created by laser light in a Compact Disc is stored in a series of tiny dents and planes (called "pits and lands") and programmed in a spiral data track into the top of polycarbonate layer. The programmed information is read by an inbuilt infrared semiconductor laser beam of $780 \mathrm{~nm}$ wavelength by a lens through the bottom of the polycarbonate layer. The reflected laser beam/s from "pits and lands" of a CD are converted into audio visual signals of the intensities of laser beams into different frequencies corresponding the "pits" dimension and remain original when reflecting off the "lands".

Indeed, the Self awareness signal passes through the infinitesimal gap or hole within the atomic structure. This changes the frequency of Self Awareness into the frequency of the received energy, which created the gap. More precisely, the Self awareness frequency converts into the frequency, which corresponds the dimension of the gap or hole in atomic structure. In other words, the Self signal becomes the signal of the object earlier perceived. This conversion and reversal to Self makes individual believe, having memory of the object. The human regular brain function is millions of times, the conversion of Self awareness into frequency of objects perceived. When this activity is hyper and Self Awareness signal is not coming back or does not reverse, the individual mental health is disturbed. Such condition of Loss of Self Awareness creates health and behavioral problems.

\subsection{Does the world around sends any information of its own natural condition?}

In the brain there is no projector, no light, no film to register external light, no screen to project the image of the physical world. Neither there is all the mechanism of a compact disc for recording and reading. Yet, the registered light frequency codes in the nucleus of lateral geniculation, when reactivated, projects faint image of the perceived world in visual cortex or primary visual cortex (V1), The image projected on the movie screen and in the brain corresponds the light reflected from the bodies.

In other words, in visual perception, the reflected light from the physical world, including humans etc; does not carry any information. Indeed, the light after reflecting does not carry any physical, physiological, chemical, biological, molecular or atomic information of the body perceived. At the instant of impingement and reflection (in light speed) the initial frequency is changed, in effect, attenuates, and changed frequency has the color attribute. Color and luminosity are the attributes of light.

\subsection{Neither there are "physical bodies" on the screen nor in the brain}

Fundamentally, the assumed memory of physical world is in true sense, self imposed "False Memory". This "false memory" held strongly or obsessively in the brain is conflict prone and creates disturbed mental conditions. It can be inferred that this memory, only for practical reason, embedded in the concrete day-to-day lives of individuals, helps organize life. The memory reactivations from $5 \mathrm{~Hz}$ up to $12 \mathrm{~Hz}$ appear between wake-sleep states. This is the condition where an individual is neither fully awake nor in deep sleep. The narration of images, called dream, are of different intensities hence the individual can sometimes narrate those images clearly and at other times he or she cannot recollect the images.

The above two states of dream images correspond to high and low intensities of brain frequencies. Between $8 \mathrm{~Hz}$ and $12 \mathrm{~Hz}$ of brain waves do carry certain intensity of image resolution, which then, the individual recollects and narrates. The low intensity of image resolution, which appears between 5 and $8 \mathrm{~Hz}$ of brain frequency, is not clearly remembered. Though the individual may express indistinct recollections of some images, which are obscure visuals, manifest just after deep sleep condition. In other case, the frequencies are near to waking state as the intensity is higher hence the possibility of remembrance. In few other cases, due to higher frequency activity, between 8 and $12 \mathrm{~Hz}$, there is ad-mixture of reactivation of previously registered visual data 
which creates a non cohesive image display or dream sequence.

\section{Discussion}

Received visual information by initial neuron signal out of an atom in the nucleus of the nerve cell, does carry with it the frequency information of externally perceived light frequencies. The conventional description of atoms is: Sodium $(\mathrm{Na})$ with Atomic Number 11 with 11 electrons and equal number of Protons including 12 Neutrons, Potassium K, Atomic Number is 19, number of Protons and Electrons are 19 and 20 Neutrons. Chlorine $\mathrm{Cl}$, Atomic Number is 17, number of Protons and Electrons are 17 and 18 Neutrons, Calcium Ca, number of Protons and Electrons are 20 with 20 Neutrons. When it is said 11, 17 or 19 and 20 Electrons, according to Quantum Mechanics, it is a "Cloud Density" corresponding to the supposed electron number. One of the atoms of sodium, potassium and calcium releases the small fraction of its own negative charge, losing partial quantity out of electron "cloud" density of that atom.

These negative charge (neuron signals) carrying the light frequency information rest in the nucleus of lateral geniculate, with the frequency codes. When the external stimuli re-activate these past codes, the brain has the faint image of that physical perception. These electromagnetic frequencies are extremely weak. Since childhood, humans are creating a self imposed embedded program through juxtapositioning descriptive audio induced (language) signals with visual light produced signal in center of brain and these reactivate as memory. These, in pure physics terms do not represent the physical world. Indeed, the physical world does not have its own means to send its own information, either in light form or audio form. A note of caution about mathematical or computational neuroscience, which leads to microchips and robotics research and it is extremely controversial by experimental use of implanting programmed microchip (or Nanochips) in patient's body or brain. Further there will be experiments on normal humans. All other research relating Psychology and Neuroscience is for pathological and clinical purpose. For all humans, research in understanding foundation of memory and the source of thought remains essential.

As a fundamental psychological function of brain, the audio-visual perception is taken as example to grasp the complexity of sense perception and sensory registration of memory. The visible spectrum of the electromagnetic band, perceptible to the human eye, is around 390 to $750 \mathrm{~nm}$ (Cecie 2005). The possible frequencies in the frequency band are in the range of 400-790 THz, which is $1.6543 \mathrm{eV}$ to $3.2672 \mathrm{eV}$. The maximum sensitivity of the human eye in the green region of the visible spectrum during the day is around 555 $\mathrm{nm}=2.2340 \mathrm{eV}$ or of $540 \mathrm{THz}=2.2333 \mathrm{eV}$. There is a mix of multiple colors and wavelengths however the human eye responds to the visible spectrum (Cut hill, 1997; Coffey, 1912; Jamieson, 2007; John, 1995; Thomas, Paris, \& Svoronos, 2005; Reproducing Visible Spectra, 2011).

$$
50 \mathrm{~Hz}=5.9958 \mathrm{e}+15 \mathrm{~nm}=2.0678 \times 10^{-13} \mathrm{eV}
$$

It is a tricky business to "convert" Hertz to voltage. Example, $50 \mathrm{~Hz}$ is a rate of change of voltage equal to 50 cycles of that voltage per second. Gamma waves are neural oscillations in human brain with a frequency between 25 to $100 \mathrm{~Hz}$, (Hughes, 2008) though $40 \mathrm{~Hz}$ is regularly observed (Ian, 1999).

$40 \mathrm{~Hz}=1.6543 \times 10^{-13} \mathrm{eV}$

In case of Sound waves, the normal sound frequency appears from $250 \mathrm{~Hz}$ to $8-12 \mathrm{kHz}$. In human, the generally accepted standard range of audible frequencies is 20 to $20,000 \mathrm{~Hz}$. The frequencies and wavelengths of spectral colors appear as: Red 400-484 THz 620-750 nm, orange 484-508 THz 590-620 nm, yellow 508-526 THz 570-590 nm, green 526-606 THz 495-570 nm, cyan 606-630 THz 476-495 nm, blue $631-668 \mathrm{THz}$ 450-475 nm and violet 668-789 THz 380-450 nm. Above frequencies are not permanent, in nature, the light reflects from millions of natural bodies and manmade structures, which makes it loose its energy content and change in frequency. The reflected light has to pass through many biophysical layers of human eye. The mechanism of visual perception by human eye is well known. The light in millions of frequencies from the 
physical environment strikes thin blanket of liquid moisture (tears) at the frontage of the eye. Passing through the material composition of human eye, the optic nerve sends the visual signals to the visual center in the back of the brain where the experience of vision occurs. The light, reflected from an object, entering the eye, focused, and transformed into electro-chemical signals arrives into the brain and construed as an image.

"Attenuation", is a decrease in property or gradual loss in intensity as energy wave flux or a beam of particles, as the distance from the source increases, due to interaction with medium, scattering, spreading in three dimensions, even without interaction. Hence, the initial energy of Neuron Spike during its trajectory to the lateral geniculation nucleus in human brain attenuates considerably i.e. decrease in the voltage field with the square of distance it travels, the incoming signal itself reaches a Zero Frequency (ZF) or in Inertia condition. If looked further and deeper, it reaches in between atomic composition inside the cell nucleus. Within the atomic composition, the incoming signal is "Energy in Rest", creating an infinitesimal gap or hole within the atomic structure. Any source of energetic activity has a condition of inactivity, called Energy in Inertia, which can be called "Energy in Zero Frequency" (EZF).

The supposed memory of physical world was tested simply by asking the individual to walk in one's own house by closed eyes, where every object is in memory held by the individual as his/her own known physical environment. The individual could not walk freely more than three steps in bedroom to bathroom or in sitting (drawing) room to kitchen or in other places. This establishes that there really is "No Information of Physical World" in the brain and it also elucidated that by open eyes, the light frequencies from each object of ones own environment invoked the previously available frequency codes, giving individual a sense of assurance of having "knowledge" of physical surrounding to move freely.

The theoretical proposition as, "The Brain Is Both Neurocomputer and Quantum Computer" by Stuart R. Hameroff (2007) and related ideas are already published (Hagan et al., 2002; Hameroff \& Penrose, 1996a; Hameroff \& Penrose, 1996b; Hameroff, Kaszniak, \& Scott, 1996). These thoughts are more about extrinsic nature and not in-depth intrinsic. With frequency codes in Zero state since early life, infancy, childhood and adult, the individual brain is encoded with billions of frequency signals created by incoming electromagnetic audio visual frequencies, also by other sensory perception. It won't be a too farfetched conclusion, if it is acknowledged that the human being is living with a self imposed program, making it a "Naturobot". The five sense organs are the programming tools.

In human beings, the neural oscillation of the brain waves are investigated by electroencephalography (EEG) called invasive detection through single-unit recordings. Intracellular neural oscillations are observed in sub threshold of the membrane potential of the waves, (Buzsaki, 2006). Yet another device, Magneto Encephalography (MEG) is used to observe synchronous activity of large numbers of neurons.

A Neuron or any nerve cell is not naturally qualified to emit subatomic phenomenon, like an electrical charge or electromagnetic pulse. An electrical or electromagnetic emission is beyond biological pulpy material of a cell, beyond chemical composition, beyond molecular composition, even beyond atomic composition.

According to basic physics mentioned earlier, the initial charge emission does in fact activate or excites other atoms in immediate vicinity, which appears as a network of Neurons activity. Whichever may be the cell, as described in five divisions of neurons within the retina are photoreceptor cells, bipolar cells, ganglion cells, horizontal cells, and amacrine cells. The basic circuitry of the retina suppose to incorporates a three-neuron chain consisting of the photoreceptor, a rod or cone, bipolar cell, and the ganglion cell and the first action potential seems to occur in the retinal ganglion cell, which is the direct path to transmit the visual information to the brain. (Purves, 2008; Ramachandran, 1998), which again must be understood as a subatomic emission out of one of the atoms in the cell composition, either out of calcium atom or potassium or sodium atom.

Samples of EEG signals show distribution of electromagnetic radiation of energy emissions. The amounts of 
energy observed are delta waves. A delta wave produced from deep sleep called slow-wave sleep is a high amplitude brain wave with a frequency of oscillation between 0-4 hertz (Walker, 1999; Kirmizialsan, 2006) and Alpha of $8-12 \mathrm{~Hz}$ detected strongest neural activity in the occipital lobe during awake and relaxed condition (Cantero et al., 2003). Theta wave is of $4-8 \mathrm{~Hz}$ (Cantero et al., 2003), and Beta is of $13-30 \mathrm{~Hz}$ and Gamma waves in 30-70 to $100 \mathrm{~Hz}$ frequency band (Berger \& Gray, 1929; Fries, 2001; Llinas \& Yarom, 1986). The brain activity or Mu waves are electromagnetic oscillations in the frequency range of $8-13 \mathrm{~Hz}$ and appear in bursts of at $9-11 \mathrm{~Hz}$. (Oberman et al., 2005; Churchland, 2011). Since most of these signals are visual and audio frequencies, the observed energy emissions are indeed electromagnetic light phenomenon. The observed data on surface level of the brain are the after effects of internal initial emissions.

Notably poor spatial resolution of Functional magnetic resonance imaging (fMRI) can directly demonstrate areas of the active brain, whereas EEG requires concentrated interpretation only to hypothesize about the areas, which are activated by a particular reaction. It is mathematically impossible to reconstruct a distinctive intracranial current source for a given EEG signal, as some currents produce potentials that cancel each other out, known as the inverse problem (Niedermeyer \& da Silva, 2004). Because voltage fields fall off with the square of distance, activity from deep sources is more difficult to detect than currents near the skull (Klein \& Thorne, 2006). EEG determines neural activity that occurs below the upper layers of the brain (the cortex) extremely inadequately. Further, none of the techniques used, can reach the source of brain activity or source of thought. Hence the results are probabilistic, statistical, meaning incongruent and always in approximations in generalized assumptions.

\section{Conclusion}

Above research concerns all humans. It would be recommended that certain basic understanding of this research is taken as mandatory in after school, then college and as specialization for after graduation is included in regular curriculum. Some part of brain research should be taken for further research, example, to discover the "stimulus" of disturbing (memory) frequencies to correct by creating new undamaging stimulus from natural environment with non conformist attitude.

The brain has only light and sound frequency codes, including stimulations from other senses, which are, indeed tiny gaps or holes in atomic structure. These are within the cellular and molecular composition in the interior of the brain. True nature of Memory is the transformation or conversions of Self Awareness signal passing through the infinitesimal gap in atomic structure created by earlier received signals into those frequencies. Indeed, in human brain, there is only one Self Awareness signal, which transforms itself, millions of times into the earlier signals.

The human brain does not have any information of the natural composition of the physical world; neither the objects of perception send any information of their own natural and original composition. The names and other description is man's invention in languages. When these two aspects are held or juxtaposed in brain, the individual thinks that he or she knows the object. Apart from practical purpose, this "memory" is not true knowledge. The brain has only light and sound frequency codes, including signals induced by other senses. A little deeper understanding is that the conventional term called "Memory" does not exist.

\section{References:}

Agrawal, N., Hamam, B. N., Magistretti, J., Alonso, A., \& Ragsdale, D. S. (1999). Persistent sodium channel activity mediates sub-threshold membrane potential oscillations and low-threshold spikes in rat entorhinal cortex layer V neurons. Journal of General Physiology, 114, 491-509.

Alonso, A., \& Llinas, R. (1989). Subthreshold Na+-dependent theta-like rhythmicity in entorhinal cortex layer II stellate cells. Nature, 342, 175-177. <http://dx.doi.org/10.1038/342175a0 >

Aurlien, H., Gjerde, I. O., Aarseth, J. H., Karlsen, B., Skeidsvoll, H., \& Gilhus, N. E. (2004). EEG background 
Katkar, N.

activity described by a large computerized database. Clinical Neurophysiology, 115(3), 665-673. <http://dx.doi.org/10.1016/j.clinph.2003.10.019>

Bauer, P., Wenner, J., Dropik, P., \& Wewerka, S. (2000). Parameters of remembering and forgetting in the transition from infancy to early childhood. Monographs of the Society for Research in Child Development, 65(4), 1-204. <http://dx.doi.org/10.1111/1540-5834.00104>

Berger, H., \& Gray, C. M. (1929). Uber das Elektroenkephalogramm des Menschen [In German]. Arch Psychiat Nervenkr, 87, 527-570. <http://dx.doi.org/10.1007/BF01797193>

Buzsaki, G. (2006). Cycle 9, The gamma buzz. Oxford.

Byrne, J. H. (2007). Plasticity: New concepts, new challenges. In H. L. Roediger, Y. Dudai, \& S. M. Fitzpatrick (Eds.), Science of memory: Concepts (pp. 77-82). New York: Oxford University Press.

Cantero, J. L., Atienza, M., Stickgold, R., Kahana, M. J., Madsen, J. R., \& Kocsis, B. (2003). Sleep-dependent theta oscillations in the human hippocampus and neocortex. Journal of Neuroscience, 23(34), 10897-10903.

Churchland, P. (2011). Braintrust. Princeton University Press.

Clevelend, E., \& Reese, E. (2008). Children remember early childhood: Long-term recall across the offset of childhood amnesia. Applied Cognitive Psychology, 22(1), 127-142. $<$ http://dx.doi.org/10.1002/acp.1359>

Coffey, P. (1912). The science of logic: An inquiry into the principles of accurate thought. Longmans.

Cuthill, I. C. (1997). Ultraviolet vision in birds. In Peter Slater, Advances in the study of behavior. Oxford, England: Academic Press.

Desmaison, D., Vincent, J. D., \& Lledo, P. M. (1999). Control of action potential timing by intrinsic sub-threshold oscillations in the olfactory bulb output neurons. Journal of Neuroscience, 19, 10727-10737.

Fivush, R., \& Schwarzmueller, A. (1999). Children remember childhood: Implications for childhood amnesia. Applied Cognitive Psychology, 12(5), 455-473. $<$ http://dx.doi.org/10.1002/(SICI)1099-0720(199810)12:5<455::AID-ACP534>3.0.CO;2-H>

Fransen, E., Alonso, A. A., \& Hasselmo, M. E. (2002). Simulations of the role of the muscarinic-activated calcium-sensitive non-specification current I (NCM) in entorhinal neuronal activity during delayed matching tasks. Journal of Neuroscience, 22, 1081-1097.

Fries, P. (2001). A mechanism for cognitive dynamics: neuronal communication through neuronal coherence. TICS, 9, 474-480.

Giocomo L. M., Zilli, E. A., Fransén, E., \& Hasselmo. M. E. (2007). Temporal frequency of sub-threshold oscillations scales with entorhinal grid cell field spacing. Science, 315, 1719 - 1722. $<$ http://dx.doi.org/10.1126/science.1139207>

Hameroff, R. S. (2007). The brain is both neurocomputer and quantum computer. Cognitive Science, 31, 1035-1045. <http://dx.doi.org/10.1080/03640210701704004>

Hameroff, S. R., \& Penrose, R. (1996a). Conscious events as orchestrated spacetime selections, Journal of Consciousness Studies, 3, 36-53.

Hameroff, S. R., \& Penrose, R. (1996b). Orchestrated reduction of quantum coherence in brain microtubules: A model for consciousness. In S. R. Hameroff, A. Kaszniak and A.C. Scott (Eds.), Towards a science of consciousness - Contributions from the 1994 Tucson. Cambridge, MA: MIT Press.

Hameroff, S. R., Kaszniak, A. W., \& Scott, A. C. (1996). Toward a science of consciousness II: The second Tucson discussions and debates. Cambridge, MA: MIT Press.

Hughes, J. R. (2008). Gamma, fast, and ultrafast waves of the brain: their relationships with epilepsy and behavior. Epilepsy Behavior, 13(1), 25-31. <http://dx.doi.org/10.1016/j.yebeh.2008.01.011>

Gold, I. (1999). Does 40-Hz oscillation play a role in visual consciousness? Consciousness and Cognition, 8(2), 186-195. <http://dx.doi.org/10.1006/ccog.1999.0399>

Jamieson, Barrie G. M. (2007). Reproductive Biology and Phylogeny of Birds. Charlottesville VA: University of Virginia. p. 128. ISBN 1578083869.

Jensen, O., \& Lisman, J. E. (2005). Hippocampal sequence-encoding driven by a cortical multi-item working 
memory buffer. Trends in Neuroscience, 26, 696-705.

Joseph, R. (2003). Emotional trauma and childhood amnesia. Consciousness and Emotion, 4(2), 151-179. $<$ http://dx.doi.org/10.1075/ce.4.2.02jos >

Khosrovani, S., Van Der Giessen, R. S., De Zeeuw C. I., \& De Jeu, M. T. G. (2007). In vivo mouse inferior olive neurons exhibit heterogeneous sub-threshold oscillations and spiking patterns. PNAS, 104, 15911-15916. <http://dx.doi.org/10.1073/pnas.0702727104>

Kirmizialsan, E., Bayraktaroglu, Z., Gurvit, H., Keskin, Y., Emre, M., \& Demiralp, T. (2006). Comparative analysis of event-related potentials during Go/NoGo and CPT: Decomposition of electrophysiological markers of response inhibition and sustained attention. Brain Research, 1104(1), 114-128. $<$ http://dx.doi.org/10.1016/j.brainres.2006.03.010>

Klein, S., \& Thorne, B. M. (2006). Biological psychology. New York: Worth.

Llinas, R., \& Yarom, Y. (1986). Oscillatory properties of guinea-pig inferior olivary neurones and their pharmacological modulation: An in vitro study. Journal of Physiology, 376, 163-182.

Llinas, R., Grace, A. A., \& Yarom, Y. (1991). In vitro neurons in mammalian cortical layer 4 exhibit intrinsic oscillatory activity in the 10 to $50 \mathrm{~Hz}$ frequency range. PNAS, 88, 897-901.

Manis, P. B., Molitor, S. C., \& Wu, H. (1999). Subthreshold oscillations generated by TTX-sensitive sodium currents in dorsal cochlear nucleus pyramidal cells. Experimental Brain Research, 153, 443-451. <http://dx.doi.org/10.1007/s00221-003-1639-6>

Morrison, C. M., \& Conway, M. A. (2010). First words and First memories. Cognition, 116(1), $23-32$. $<$ http://dx.doi.org/10.1016/j.cognition.2010.03.011>

Niedermeyer, E., \& da Silva, F. L. (2004). Electroencephalography: Basic principles, clinical applications, and related fields. Lippincot Williams \& Wilkins.

Oberman, L. M., Hubbard, E. M., McCleery, J. P., Altschuler, E. L., Ramachandran, V. S., \& Pineda, J. A. (2005) EEG evidence for mirror neuron dysfunction in autism spectrum disorders. Cognitive Brain Research, 24(2), 190-198. <http://dx.doi.org/10.1016/j.cogbrainres.2005.01.014>

Pedroarena, C., \& Llinas, R. (1997). Dendritic calcium conductances generate high frequency oscillation in thalamocortical neurons. PNAS, 94, 724-728. <http://dx.doi.org/10.1073/pnas.94.2.724>

Phelps, E. (2004). Human emotion and memory: Interactions of the amygdala and hippocampal complex. Current Opinion in Neurobiology, 14(2), 198-202. <http://dx.doi.org/10.1016/j.conb.2004.03.015>

Purves, D., Augustine, G. J., Fitzpatrick, D., Hall, W. C., LaMantia, A., McNamara, J. O., \& White, L. E. (2008). Neuroscience ( $4^{\text {th }}$ ed.) Sunderland, MA: Sinauer Associates.

Ramachandran, V. S., \& Blakeslee, S. (1998). Phantoms in the brain: Probing the mysteries of the human mind. William Morrow \& Company.

Robinson-Riegler, G., \& Robinson-Riegler, B. (2012). Cognitive psychology: Applying the science of the mind $\left(3^{\text {rd }}\right.$ ed.). Boston, MA: Pearson education.

Schmitz, D., Gloveli, T., Behr. J., Dugladze, T., \& Heinemann, U. (1998). Subthreshold membrane potential oscillations in neurons of deep layers of the entorhinal cortex. Neuroscience, 85, 999-1004. $<$ http://dx.doi.org/10.1016/S0306-4522(98)00113-4>

Schwarzel. M., \& Mulluer, U. (2006). Dynamic memory networks. Cellular and Molecular Life Science, 63, 989-998.

ScienceDaily. (2011). Mimicking the brain -- In silicon: New computer chip models how neurons communicate with each other at synapses.

Simcock, G., \& Hayne, H. (2000). Age-related changes in verbal and nonverbal memory during early childhood. Psychological Science, 9(2), 55-58.

Starr, C. (2005). Biology: Concepts and applications. Thomson Brooks/Cole.

Bruno, T. J., Paris, D. N., \& Svoronos, C. R. C. (2005). Handbook of fundamental spectroscopic correlation charts. CRC Press.

Walker, P. (1999). Chambers dictionary of science and technology. Edinburgh: Chambers. 
Katkar, N. 